\title{
Potential of autotaxin as a diagnostic marker and therapeutic target for itch of cholestasis
}

New findings show that increased serum autotaxin activity is specific to itch of cholestasis and that autotaxin activity corresponds to the effect achieved by the various different treatments available.

"We previously identified lysophosphatidic acid (LPA) and the LPA-forming enzyme autotaxin as candidate inducers of cholestatic itch and found that itch intensity correlated with serum autotaxin activity," explains Ulrich Beuers, who headed up the study with Ronald Oude Elferink. The next step for their team was to see if increased serum autotaxin activity was specific to cholestatic itch and look at the effect of itch therapies on autotaxin activity.

At a cut-off value of $8.5 \mathrm{nmol} / \mathrm{ml} / \mathrm{min}$, autotaxin activity had a sensitivity, specificity and positive predictive value for cholestatic liver disease of $71 \%, 91 \%$ and $70 \%$, respectively, when compared with itch of uraemia, Hodgkin's disease or

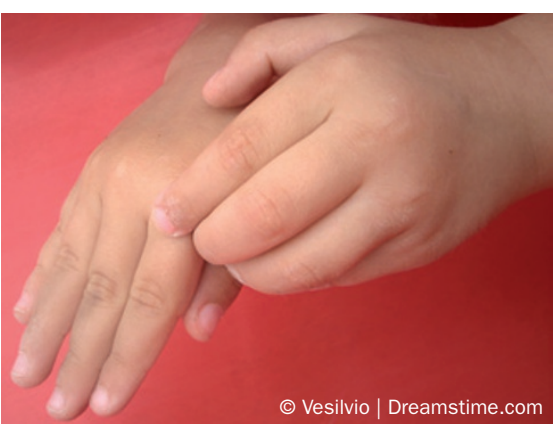

intensity and autotaxin activity in patients who gained no relief with colesevelam. "In vitro tests show that the anti-itch effect of rifampicin is explained, at least in part, by Pregnane X Receptor-dependent transcriptional inhibition of autotaxin expression," says Beuers. Itch intensity and autotaxin activity were also diminished moderately by Molecular Adsorbents Recirculation System (MARS) therapy and markedly by nasobiliary drainage.

The team argue that their findings atopic dermatitis. As such, autotaxin could potentially serve as a novel diagnostic marker for use in patients with itch of unknown origin and help target therapy in those with more than two potentially itch-inducing conditions.

Bile acid sequestrants are the first-line therapy for cholestatic itch, but in this study colesevelam had a minimal effect on itch intensity and autotaxin activity. Rifampicin, the recommended secondline therapy, moderately diminished itch question a direct causal role for bile salts in itch and support the potential of autotaxin inhibitors and LPA receptor blockers as future treatments for cholestatic itch.

Natalie J. Wood

Original article Kremer, A. E. et al. Serum autotaxin is and responds to therapeutic interventions. Hepatology doi:10.1002/hep.25748 increased in pruritis of cholestasis, but not of other origins 